IJMMS 27:10 (2001) 621-630

PII. S0161171201004884

http://ijmms.hindawi.com

(c) Hindawi Publishing Corp.

\title{
ON SEPARATION AXIOMS IN INTUITIONISTIC TOPOLOGICAL SPACES
}

\author{
SADIK BAYHAN and DOǦAN ÇOKER
}

(Received 21 March 2000)

\begin{abstract}
The purpose of this paper is to investigate several types of separation axioms in intuitionistic topological spaces, developed by Çoker (2000). After giving some characterizations of $T_{1}$ and $T_{2}$ separation axioms in intuitionistic topological spaces, we give interrelations between several types of separation axioms and some counterexamples.
\end{abstract}

2000 Mathematics Subject Classification. 54A99.

1. Introduction. After the introduction of the concept of a fuzzy set by Zadeh [15], Atanassov [1, 2] has introduced the concept of intuitionistic fuzzy set. Later Çoker et al. $[4,5,8]$ have defined intuitionistic fuzzy topological spaces, intuitionistic sets, and intuitionistic topological spaces in [6, 9, 12].

2. Preliminaries. First we present the fundamental definitions (see Çoker [4]).

DEFINITION 2.1 (see [4]). Let $X$ be a nonempty fixed set. An intuitionistic fuzzy set (IS for short) $A$ is an object having the form $A=\left\langle X, A_{1}, A_{2}\right\rangle$, where $A_{1}$ and $A_{2}$ are subsets of $X$ satisfying $A_{1} \cap A_{2}=\varnothing$. The set $A_{1}$ is called the set of members of $A$, while $A_{2}$ is called the set of nonmembers of $A$.

DEFINITION 2.2 (see [4]). Let $X$ be a nonempty set and let the IS's $A$ and $B$ be in the form $A=\left\langle X, A_{1}, A_{2}\right\rangle, B=\left\langle X, B_{1}, B_{2}\right\rangle$, respectively. Furthermore, let $\left\{A_{i}: i \in J\right\}$ be an arbitrary family of IS's in $X$, where $A_{i}=\left\langle X, A_{i}^{(1)}, A_{i}^{(2)}\right\rangle$. Then

(a) $A \subseteq B$ if and only if $A_{1} \subseteq B_{1}$ and $A_{2} \supseteq B_{2}$;

(b) $A=B$ if and only if $A \subseteq B$ and $B \subseteq A$;

(c) $\bar{A}=\left\langle X, A_{2}, A_{1}\right\rangle$;

(d) $\cup A_{i}=\left\langle X, \cup A_{i}^{(1)}, \cap A_{i}^{(2)}\right\rangle$;

(e) $\cap A_{i}=\left\langle X, \cap A_{i}^{(1)}, \cup A_{i}^{(2)}\right\rangle$;

(f) []$A=\left\langle X, A_{1}, A_{1}^{c}\right\rangle$;

(g) \langle\rangle $A=\left\langle X, A_{2}^{c}, A_{2}\right\rangle$;

(h) $\varnothing_{\sim}=\langle X, \varnothing, X\rangle ; \underset{\sim}{X}=\langle X, X, \varnothing\rangle$.

Let $X$ be a nonempty set, $p \in X$ a fixed element in $X$, and let $A=\left\langle X, A_{1}, A_{2}\right\rangle$ be an IS. The IS $p$ defined by $p=\left\langle X,\{p\},\{p\}^{c}\right\rangle$ is called an intuitionistic point (IP for short) in $X$. The IS $\underset{\sim}{p}=\left\langle\varnothing,\{p\}^{c}\right\rangle$ is called a vanishing intuitionistic point (VIP for short) in $X$. The IS $p$ is said to be contained in $A$ ( $p \in A$ for short) if and only if $p \in A_{1}$, and similarly, $\underset{\approx}{\tilde{p}}$ is said to be contained in $A(\underset{\approx}{\tilde{p}} \in A$ for short $)$ if and only if $p \notin A_{2}$. For a 
given IS $A$ in $X$, we may write

$$
A=(\cup \underset{\sim}{\sim} \underset{\sim}{p} \underset{\sim}{p} \in A\}) \cup(\underset{\approx}{\cup} \underset{\approx}{p} \underset{\approx}{p} \in A\}),
$$

(cf. [9]) and whenever $A$ is not a proper IS (i.e., if $A$ is not of the form $A=\left\langle X, A_{1}, A_{2}\right\rangle$, where $\left.A_{1} \cup A_{2} \neq X\right)$, then $A=\cup\{p: p \in A\}$ follows. In general, any IS $A$ in $X$ can be written in the form $A=A \cup \underset{\approx}{A}$, where $\underset{\sim}{A}=\cup\{\underset{\sim}{p}: \underset{\sim}{p} \in A\}$ and $\underset{\approx}{A}=\cup\{\underset{\widetilde{c}}{p}: \underset{\approx}{p} \in A\}$. Furthermore it is easy to show that, if $A=\left\langle X, A_{1}, A_{2}\right\rangle$, then $\underset{\sim}{A}=\left\langle\underset{\tilde{X}}{\widetilde{X}}, A_{1}, A_{1}^{\tilde{\widetilde{c}}}\right\rangle$ and $\underset{\approx}{\approx}=$ $\left\langle X, \varnothing, A_{2}\right\rangle$ (cf. [4, 7]).

Definition 2.3 (see [4]). Let $X$ and $Y$ be two nonempty sets and $f: X \rightarrow Y$ a function, $B=\left\langle Y, B_{1}, B_{2}\right\rangle$ an IS in $Y$ and $A=\left\langle X, A_{1}, A_{2}\right\rangle$ an IS in $X$. Then the preimage of $B$ under $f$, denoted by $f^{-1}(B)$, is the IS in $X$ defined by $f^{-1}(B)=\left\langle X, f^{-1}\left(B_{1}\right)\right.$, $\left.f^{-1}\left(B_{2}\right)\right\rangle$, and the image of $A$ under $f$, denoted by $f(A)$, is the IS in $Y$ defined by $f(A)=\left\langle Y, f\left(A_{1}\right), f_{-}\left(A_{2}\right)\right\rangle$ where $f_{-}\left(A_{2}\right)=\left(f\left(A_{2}^{c}\right)\right)^{c}$.

You may find the fundamental properties of preimages and images in [4].

DEFINITION 2.4 (see [6]). An intuitionistic topology (IT for short) on a nonempty set $X$ is a family $\tau$ of IS's in $X$ containing $\underset{\sim}{\varnothing} \underset{\sim}{X}$ and closed under finite infima and arbitrary suprema. In this case the pair $(X, \tau)$ is called an intuitionistic topological space (ITS for short) and any IS in $\tau$ is known as an intuitionistic open set (IOS for short) in $X$. The complement $\bar{A}$ of an IOS $A$ in an ITS $(X, \tau)$ is called an intuitionistic closed set (ICS for short) in $X$.

Let $(X, \tau)$ be an ITS on $X$. Then, we can also construct several other ITS's on $X$ in the following way: $\tau_{0,1}=\{[] G: G \in \tau\}$ and $\tau_{0,2}=\{\langle\rangle G: G \in \tau\}$. Furthermore,

$$
\tau_{1}=\left\{G_{1}: G=\left\langle X, G_{1}, G_{2}\right\rangle \in \tau\right\}, \quad \tau_{2}=\left\{G_{2}^{c}: G=\left\langle X, G_{1}, G_{2}\right\rangle \in \tau\right\}
$$

are topological spaces in $X$ (cf. [6]).

DefinITION 2.5. Let $A$ and $B$ be two IS's on $X$ and $Y$, respectively. Then the product intuitionistic set (PIS for short) of $A$ and $B$ on $X \times Y$ is defined by $U \times V=\left\langle(X, Y), A_{1} \times\right.$ $\left.B_{1},\left(A_{2}^{c} \times B_{2}^{c}\right)^{c}\right\rangle$, where $A=\left\langle X, A_{1}, A_{2}\right\rangle$ and $B=\left\langle Y, B_{1}, B_{2}\right\rangle$.

If $(X, \tau)$ and $(Y, \Phi)$ are ITS's, then the product topology $\tau \times \Phi$ on $X \times Y$ is the IT generated by the base $\mathscr{B}=\{A \times B: A \in \tau, B \in \Phi\}$. This is so, because, if $A \times B, C \times D \in \mathscr{B}$, then $(A \times B) \cap(C \times D)=(A \cap C) \times(B \cap D)$. Let $A \in \tau, B \in \Phi$, and $A=\left\langle X, A_{1}, A_{2}\right\rangle$, $B=\left\langle Y, B_{1}, B_{2}\right\rangle$. Then we have $\pi_{1}^{-1}(A)=\left\langle(x, y), A_{1} \times Y, A_{2} \times Y\right\rangle=A \times \underset{\sim}{Y}, \pi_{2}^{-1}(B)=$ $\left\langle(X, Y), X \times B_{1}, X \times B_{2}\right\rangle=\underset{\sim}{X} \times B$, and

$$
\begin{aligned}
\pi_{1}^{-1}(A) \cap \pi_{2}^{-1}(B) & =(A \times \underset{\sim}{Y}) \cap \underset{\sim}{X} \times B) \\
& =\left\langle(X, Y),\left(A_{1} \times Y\right) \cap\left(X \times B_{1}\right),\left(A_{2} \times Y\right) \cup\left(X \times B_{2}\right)\right\rangle \\
& =\left\langle(X, Y), A_{1} \times B_{1},\left(A_{2} \times Y\right) \cup\left(X \times B_{2}\right)\right\rangle \\
& =\left\langle(X, Y), A_{1} \times B_{1},\left(A_{2}^{c} \times B_{2}^{c}\right)^{c}\right\rangle=A \times B .
\end{aligned}
$$


The definition of "neighborhoods" of IP's and VIP's can be found in Coşkun and Çoker [9] and "continuous function" between ITS's can be found in Çoker [6].

LEMMA 2.6. The projections $\pi_{1}: X \times Y \rightarrow X, \pi_{2}: X \times Y \rightarrow Y, \pi_{1}(x, y)=x, \pi_{2}(x, y)=$ $y$ are continuous.

Proof. Let $A \in \tau$, then $\pi_{1}^{-1}(A)=\left\langle(x, y), \pi_{1}^{-1}\left(A_{1}\right), \pi_{1}^{-1}\left(A_{2}\right)\right\rangle$. Thus we have $\pi_{1}^{-1}(A)$ $=\left\langle(x, y), A_{1} \times Y, A_{2} \times Y\right\rangle=A \times \underset{\sim}{Y}$, that is, $\pi_{1}$ is continuous.

In other words, the product topology $\tau \times \Phi$ on $X \times Y$ is indeed the initial topology on $X \times Y$ with respect to the projections $\pi_{1}: X \times Y \rightarrow X$ and $\pi_{2}: X \times Y \rightarrow Y$. Here the subbase $\left\{\pi_{1}^{-1}(A), \pi_{2}^{-1}(B): A \in \tau, B \in \Phi\right\}$ generates this product topology and the base $\mathscr{B}$ is given by

$$
\mathscr{B}=\left\{\pi_{1}^{-1}(A) \cap \pi_{2}^{-1}(B): A \in \tau, B \in \Phi\right\}=\{A \times B: A \in \tau, B \in \Phi\} .
$$

DeFinItion 2.7. Given the nonempty set $X$, we define the diagonal $\Delta_{x}$ as the following IS in $X \times X$ :

$$
\Delta_{x}=\left\langle\left(x_{1}, x_{2}\right),\left\{\left(x_{1}, x_{2}\right): x_{1}=x_{2}\right\},\left\{\left(x_{1}, x_{2}\right): x_{1} \neq x_{2}\right\}\right\rangle .
$$

Notice that, if $X$ and $Y$ are two nonempty sets and $(p, q) \in X \times Y$ a fixed element in $X \times Y$, then $(p, q) \sim$ is contained in $U \times V((p, q) \sim \in U \times V$ for short) if and only if $(p, q) \in U_{1} \times V_{1}$, and $(p, q) \approx$ is contained in $U \times V((p, q) \approx \in \times V$ for short $)$ if and only if $(p, q) \notin\left(U_{2}^{c} \times V_{2}^{c}\right)^{c}$, or equivalently $(p, q) \in U_{2}^{c} \times V_{2}^{c}$.

DeFinItion 2.8. Let $X, Y$ be two nonempty sets and $f: X \rightarrow Y$ a function. The graph of $f$, denoted by $\mathrm{GR}(f)$, is defined as the following IS in $X \times Y$ :

$$
\operatorname{GR}(f)=\left\langle(x, y),\{(x, f(x)): x \in X\},\{(x, f(x)): x \in X\}^{c}\right\rangle .
$$

3. Separation axioms in intuitionistic topological spaces. In this section, we present $T_{1}$ and $T_{2}$ separation axioms in ITS's. The separation axioms $T_{1}$ and $T_{2}$ presented here have certain similarities to those in Bayhan and Çoker [3].

DEFINITION 3.1. Let $(X, \tau)$ be an ITS, $(X, \tau)$ is said to be
(a) $T_{1}(i) \Leftrightarrow \forall x, y \in X(x \neq y) \exists U, V \in \tau$ such that $\underset{\sim}{x} \in U, \underset{\sim}{ } \notin U$, and $y \in V$, $x \notin V$ (cf. [3, 14]);
(b) $T_{1}(i i) \Leftrightarrow \forall x, y \in X(x \neq y) \exists U, V \in \tau$ such that $\underset{\approx}{x} \in U, \underset{\approx}{y} \notin U$, and $\underset{\approx}{y} \in V$, $\underset{\sim}{x} \notin \underset{\sim}{x} \in V$ (cf. [3, 14]);

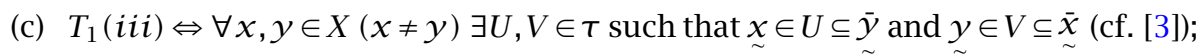

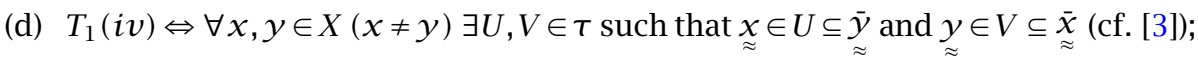
(e) $T_{1}(v) \Leftrightarrow \forall x, y \in X(x \neq y) \exists U, V \in \tau$ such that $\underset{\sim}{y} \notin U$ and $\underset{\sim}{x} \notin V$ (cf. [3]);
(f) $T_{1}(v i) \Leftrightarrow \forall x, y \in X(x \neq y) \exists U, V \in \tau$ such that $\underset{\approx}{y} \notin U$ and $\underset{\approx}{x} \notin V$ (cf. [3]);
(g) $T_{1}$ (vii) $\Leftrightarrow \forall x \in X, \underset{\sim}{x}$ is $\tau$-closed;
(h) $T_{1}$ (viii) $\Leftrightarrow \forall x \in X, \underset{\approx}{x}$ is $T$-closed. 
THEOREM 3.2. Let $(X, \tau)$ be an ITS, then the following implications are valid:

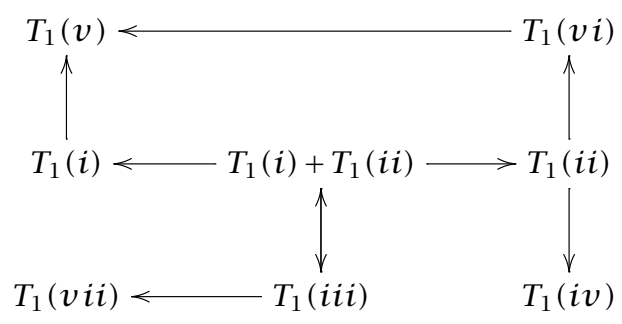

Proof. The proof is obvious.

CountereXAmple 3.3. Let $X=\{a, b, c\}$ and define the IT $\tau=\{\varnothing, \underset{\sim}{\varnothing}, A, B, C, D$, $E, F, G\}$, where $A=\langle X,\{a, c\}, \varnothing\rangle, B=\langle X,\{b\}, \varnothing\rangle, C=\langle X,\{a\}, \varnothing\rangle, D=\langle X,\{c\}, \varnothing\rangle$, $E=\langle X,\{a, b\}, \varnothing\rangle, F=\langle X,\{b, c\}, \varnothing\rangle, G=\langle X, \varnothing, \varnothing\rangle$. Then $(X, \tau)$ is $T_{1}(i)$, but not $T_{1}(i i)$.

COUNTEREXAMPLE 3.4. Let $X=\{a, b\}$ and define the IT $\tau=\{\underset{\sim}{\varnothing} \underset{\sim}{X}, A, B\}$ on $X$, where $A=\langle X, \varnothing,\{a\}\rangle, B=\langle X, \varnothing,\{b\}\rangle$. Then it is clear that $(X, \tau)$ is $\tilde{T}_{1}(v)$, but not $T_{1}(i)$.

COUNTEREXAMPLe 3.5. Let $X=\{a, b, c\}$ and define the IT $\tau=\{\underset{\sim}{\varnothing} \underset{\sim}{X}, A, B, C, D, E, F\}$ on $X$, where $A=\langle X, \varnothing,\{a, b\}\rangle, B=\langle X,\{c\},\{a, b\}\rangle, C=\langle X, \varnothing,\{b, c\}\rangle, D=\langle X,\{c\},\{b\}\rangle$, $E=\langle X,\{a, c\},\{b\}\rangle, F=\langle X, \varnothing,\{b\}\rangle$. Then $(X, \tau)$ is $T_{1}(v i)$, but not $T_{1}(i i)$.

CountereXAmple 3.6. Let $X=\{a, b, c\}$ and define the IS's $A=\langle X,\{a\},\{c\}\rangle, B=$ $\langle X,\{b\},\{a\}\rangle, C=\langle X,\{a\},\{b, c\}\rangle, D=\langle X, \varnothing,\{b\}\rangle, E=\langle X,\{a, b\}, \varnothing\rangle, F=\langle X, \varnothing,\{a, c\}\rangle$, $G=\langle X, \varnothing,\{b, c\}\rangle, H=\langle X,\{a\}, \varnothing\rangle, K=\langle X,\{a\},\{b\}\rangle$. Let $\tau$ denote the IT on $X$ generated by the subbase $S=\{A, B, C, D, E, F, G, H, K\}$. Then $(X, \tau)$ is clearly $T_{1}(i v)$, but not $T_{1}$ (iii).

COUNTEREXAMPLE 3.7. Let $X=\{a, b, c, d\}$ and consider the family $\tau=\{\underset{\sim}{\varnothing} \underset{\sim}{X}, A, B$, $C, D, E, F, G\}$, where $A=\langle X,\{a\}, \varnothing\rangle, B=\langle X,\{b\},\{\varnothing\}\rangle, C=\langle X,\{c\}, \varnothing\rangle, D=\langle X,\{a, b\}, \varnothing\rangle$, $E=\langle X,\{b, c\}, \varnothing\rangle, F=\langle X,\{a, b, c\}, \varnothing\rangle, G=\langle X, \varnothing, \varnothing\rangle$. Then the ITS $(X, \tau)$ is $T_{1}(v)$, but not $T_{1}(v i)$.

COUNTEREXAMPLE 3.8. Let $X=\{a, b, c\}$ and consider the family $\tau=\{\underset{\sim}{\varnothing} \underset{\sim}{X}, A, B, C$, $D, E, F, G, H, K\}$, where $A=\langle X,\{a\},\{c\}\rangle, B=\langle X,\{b\}, \varnothing\rangle, C=\langle X,\{c\}, \varnothing\rangle, D=\langle X,\{a, b\}$, $\varnothing\rangle, E=\langle X,\{a, c\}, \varnothing\rangle, F=\langle X,\{b, c\}, \varnothing\rangle, G=\langle X, \varnothing,\{c\}\rangle, H=\langle X, \varnothing, \varnothing\rangle, K=\langle X,\{a\}, \varnothing\rangle$. Then the ITS $(X, \tau)$ on $X$ is $T_{1}(i)$, but not $T_{1}(i i i)$.

COUNTEREXAMPLE 3.9. Let $X=\{a, b, c\}$ and consider the family $\tau=\{\underset{\sim}{\varnothing} \underset{\sim}{X}, A, B, C$, $D, E, F, G\}$, where $A=\langle X,\{a, c\}, \varnothing\rangle, B=\langle X,\{b, c\}, \varnothing\rangle, C=\langle X,\{b\}, \varnothing\rangle, D=\langle X,\{a, b\}, \varnothing\rangle$, $E=\langle X,\{c\}, \varnothing\rangle, F=\langle X,\{a\}, \varnothing\rangle, G=\langle X, \varnothing, \varnothing\rangle$. Then the ITS $(X, \tau)$ on $X$ is $T_{1}(i v)$, but not $T_{1}(i i)$. 
COUNTEREXAMPLE 3.10 (see [6]). Let $X=\mathbb{N}^{+}$and consider the IS's $A_{n}$ given below:

$$
\begin{aligned}
& A_{1}=\langle X,\{2,3,4, \ldots\}, \varnothing\rangle, \\
& A_{2}=\langle X,\{3,4,5, \ldots\},\{1\}\rangle, \\
& A_{3}=\langle X,\{4,5,6, \ldots\},\{1,2\}\rangle, \\
& A_{n}=\langle X,\{n+1, n+2, n+3, \ldots\},\{1,2,3, \ldots, n-1\}\rangle \quad(n \geq 2) .
\end{aligned}
$$

Then $\tau=\{\underset{\sim}{\varnothing} \underset{\sim}{X}\} \cup\left\{A_{n}: n=1,2,3, \ldots\right\}$ is an IT on $X$. Clearly $(X, \tau)$ is $T_{1}(v i)$, but not $T_{1}(i i)$.

Proposition 3.11. Let $(X, \tau)$ be an ITS. Then

(a) $(X, \tau)$ is $T_{1}(i)$ if and only if $\left(X, \tau_{1}\right)$ is $T_{1}$.

(b) $(X, \tau)$ is $T_{1}$ (ii) if and only if $\left(X, \tau_{2}\right)$ is $T_{1}$.

(c) $(X, \tau)$ is $T_{1}(i)$ if and only if $\left(X, \tau_{0,1}\right)$ is $T_{1}(i)$.

(d) $(X, \tau)$ is $T_{1}\left(\right.$ ii) if and only if $\left(X, \tau_{0,2}\right)$ is $T_{1}(i i)$.

DEFINITION 3.12. Let $(X, \tau)$ be an ITS. $(X, \tau)$ is said to be

(a) $T_{2}(i) \Leftrightarrow \forall x, y \in X(x \neq y) \exists U, V \in \tau$ such that $\underset{\sim}{x} \in U, \underset{\sim}{y} \in V$, and $U \cap V=\underset{\sim}{\varnothing}$ (cf. [3, 13]);

(b) $T_{2}(i i) \Leftrightarrow \forall x, y \in X(x \neq y) \exists U, V \in \tau$ such that $\underset{\sim}{x} \in U, \underset{\approx}{y} \in V$, and $U \cap V=\underset{\sim}{\varnothing}$ (cf. [3, 13]);

(c) $T_{2}$ (iii) $\Leftrightarrow \forall x, y \in X(x \neq y) \exists U, V \in \tau$ such that $\underset{\sim}{x} \in U, \underset{\sim}{y} \in V$, and $U \subseteq \bar{V}$ (cf. $[3,10])$

(d) $T_{2}(i v) \Leftrightarrow \forall x, y \in X(x \neq y) \exists U, V \in \tau$ such that $\underset{\approx}{x} \in U, \underset{\approx}{y} \in V$, and $U \subseteq \bar{V}$ (cf. $[3,10]) ;$

(e) $T_{2}(v) \Leftrightarrow \forall x, y \in X(x \neq y) \exists U, V \in \tau$ such that $\underset{\sim}{x} \in U \subseteq \underset{\sim}{\bar{y}}, \underset{\sim}{y} \in V \subseteq \underset{\sim}{\bar{x}}$, and $U \cap V=\varnothing$ (cf. [3, 11]);

(f) $T_{2}(v i) \Leftrightarrow \forall x, y \in X(x \neq y) \exists U, V \in \tau$ such that $\underset{\sim}{x} \in U \subseteq \underset{\sim}{\bar{y}}, \underset{\sim}{y} \in V \subseteq \underset{\sim}{\bar{x}}$, and $U \cap V=\varnothing$ (cf. [3, 11]);

(g) $T_{2}(v i i) \Leftrightarrow \Delta_{X}$ is an ICS in the product ITS $\left(X \times X, \tau_{X \times X}\right)$.

THEOREM 3.13. Let $(X, \tau)$ be an ITS. Then the following implications are valid:

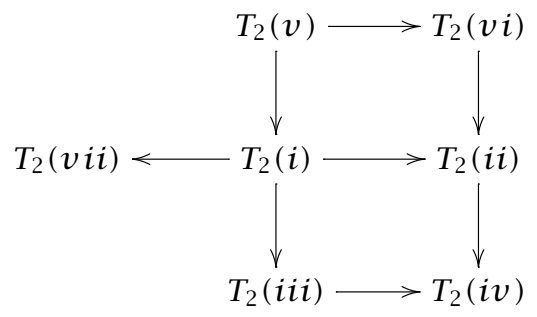

Proof. We prove only the case $T_{2}(i) \Rightarrow T_{2}$ (vii). We must see that $\bar{\Delta}_{X}$ is an IOS in $\left(X \times X, \tau_{X \times X}\right)$. Let $(x, y)_{\sim} \in \bar{\Delta}_{X}$. This means that $(x, y) \in\{(x, y): x \neq y\}$, that is, $x \neq y$. Since $(X, \tau)$ is $T_{2}(i)$, there exist $U, V \in \tau$ such that $\underset{\sim}{x} \in U, \underset{\sim}{ } \in V$, and $U \cap V=\underset{\sim}{\varnothing}$. Now in this case we have $(x, y)_{\sim} \in U \times V \subseteq \bar{\Delta}_{X}$. Indeed, from $x \in \tilde{U}_{1}$ and $y \in V_{1}$ we get 
$(x, y) \in U_{1} \times V_{1}$, that is, $(x, y)_{\sim} \in U \times V$. We also know that $U \times V \subseteq \bar{\Delta}_{X} \Leftrightarrow U_{1} \times V_{1} \subseteq$ $\{(x, y): x \neq y\}$ and $\left(U_{2}^{c} \times V_{2}^{c}\right)^{c} \supseteq\{(x, y): x=y\}$. If $\left(y_{1}, y_{2}\right) \in U_{1} \times V_{1}$, then $y_{1} \in U_{1}$, $y_{2} \in V_{1} \Rightarrow y_{1} \neq y_{2} \Rightarrow\left(y_{1}, y_{2}\right) \in\{(x, y): x \neq y\}$ follows. Thus the first inclusion is true. For the second, $\left(y_{1}, y_{2}\right) \in U_{2}^{c} \times V_{2}^{c} \Rightarrow y_{1} \in U_{2}^{c}$ and $y_{2} \in V_{2}^{c} \Rightarrow y_{1} \neq y_{2}$, that is, we have $U_{2}^{c} \times V_{2}^{c} \subseteq\{(x, y): x \neq y\}$. Thus we see that $\left(y_{1}, y_{2}\right) \in\{(x, y): x=y\}$. The second inclusion is true, too. Now since

$$
\bar{\Delta}_{X}=\bigcup_{\left(y_{1}, y_{2}\right) \sim \in \bar{\Delta}_{x}}\left(y_{1}, y_{2}\right)_{\sim},
$$

it follows from the fact that $\bar{\Delta}_{X}$ is not a proper IS, that $\bar{\Delta}_{X}$ is an IOS in $(X \times X)$, that is, $(X, \tau)$ is $T_{2}(v i i)$.

COUNTEREXAMPLE 3.14. Let $X=\{a, b\}$ and consider the family $\tau=\{\underset{\sim}{\varnothing} \underset{\sim}{X}, A, B\}$ on $X$, where $A=\langle X, \varnothing,\{b\}\rangle, B=\langle X, \varnothing,\{a\}\rangle$. Then the ITS $(X, \tau)$ on $X$ is $T_{2}(i i)$, but not $T_{2}(i)$.

COUnTEREXAmple 3.15. Let $X=\{a, b, c\}$ and define the IS's $A=\langle X, \varnothing,\{b, c\}\rangle$, $B=\langle X,\{b\},\{a\}\rangle, C=\langle X,\{a\},\{c\}\rangle$, and $D=\langle X, \varnothing,\{a, b\}\rangle$. Let $\tau$ denote the IT on $X$ generated by the subbase $S=\{A, B, C, D\}$. Then $(X, \tau)$ is $T_{2}(i v)$, but not $T_{2}$ (iii)

COUNTEREXAMPLE 3.16. Let $X=\{a, b, c\}$ and consider the family $\tau=\{\underset{\sim}{\underset{\sim}{\varnothing}} \underset{\sim}{X}, A, B, C$, $D, E, F, G, H, K, L, M\}$ on $X$, where $A=\langle X, \varnothing,\{b\}\rangle, B=\langle X, \varnothing,\{a, c\}\rangle, C=\langle X,\{a\},\{b, c\}\rangle$, $D=\langle X, \varnothing,\{a\}\rangle, E=\langle X, \varnothing,\{a, b\}\rangle, F=\langle X, \varnothing,\{c\}\rangle, G=\langle X,\{a\},\{c\}\rangle, H=\langle X,\{a\}, \varnothing\rangle$, $K=\langle X,\{a\},\{b\}\rangle, L=\langle X, \varnothing,\{b, c\}\rangle$, and $M=\langle X, \varnothing, \varnothing\rangle$. Then the ITS $(X, \tau)$ on $X$ is $T_{2}(v i)$, but not $T_{2}(v)$.

CountereXAmple 3.17. Let $X=\{a, b, c, d\}$ and define the IS's $A=\langle X,\{a\},\{b\}\rangle$, $B=\langle X,\{b\},\{a, d\}\rangle, C=\langle X,\{b\},\{c\}\rangle, D=\langle X,\{c\},\{a, b\}\rangle, E=\langle X,\{a\},\{d\}\rangle, F=\langle X,\{d\}$, $\{a\}\rangle, G=\langle X,\{b\},\{d\}\rangle, H=\langle X,\{d\},\{b\}\rangle, K=\langle X,\{c\},\{d\}\rangle, L=\langle X,\{d\},\{c\}\rangle, M=$ $\langle X,\{a\},\{c\}\rangle$, and $N=\langle X,\{c\},\{a\}\rangle$. Let $\tau$ denote the IT on $X$ generated by the subbase $S=\{A, B, C, D, E, F, G, H, K, L, M, N\}$. Then $(X, \tau)$ is $T_{2}(i i i)$, but not $T_{2}(i)$.

COUNTEREXAMPLE 3.18. Let $X=\{a, b\}$ and consider the family $\tau=\{\underset{\sim}{\varnothing} \underset{\sim}{X}, A, B\}$ on $X$, where $A=\langle X,\{b\}, \varnothing\rangle, B=\langle X, \varnothing,\{b\}\rangle$. Then the ITS $(X, \tau)$ on $X$ is $T_{2}(i v)$, but not $T_{2}(i i)$.

COUNTEREXAMPLE 3.19. We consider the IT on $X$ as in Counterexample 3.15. $(X, \tau)$ is $T_{2}(i v)$, but not $T_{2}(i)$.

COUNTEREXAMPLe 3.20. We consider the ITS on $X$ as in Counterexample 3.14. $(X, \tau)$ is $T_{2}(i i)$, but not $T_{2}(v)$.

Proposition 3.21. Let $(X, \tau)$ be an ITS. Then

(a) $(X, \tau)$ is $T_{2}(i) \Rightarrow\left(X, \tau_{1}\right)$ is $T_{2}$.

(b) $(X, \tau)$ is $T_{2}(i i) \Rightarrow\left(X, \tau_{2}\right)$ is $T_{2}$.

Proposition 3.22. Let $(X, \tau)$ be an ITS. Then

(a) $(X, \tau)$ is $T_{2}(i) \Rightarrow\left(X, \tau_{0,1}\right)$ is $T_{2}(i)$.

(b) $(X, \tau)$ is $T_{2}(i i) \Rightarrow\left(X, \tau_{0,2}\right)$ is $T_{2}(i i)$. 
THEOREM 3.23. Let $(X, \tau)$ be an ITS. Then the following implications are valid:
(a) $T_{2}($ i $) \Rightarrow T_{1}($ iii).
(b) $T_{2}$ (ii) $\Rightarrow T_{1}(\mathrm{ii})$.
(c) $T_{2}$ (iii) $\Rightarrow T_{1}($ iii).
(d) $T_{2}(i v) \Rightarrow T_{1}(i v)$.
(e) $T_{2}(v) \Rightarrow T_{1}(\mathrm{iii})$.
(f) $T_{2}(v i) \Rightarrow T_{1}(v i)$.

Proof. The proof is obvious.

Proposition 3.24. Let $(X, \tau)$ be $T_{2}(i)$. Then every intuitionistic point $\underset{\sim}{x}$ is the intersection of all the intuitionistic closed neighborhoods of $\underset{\sim}{x}$.

Proof. Let $(X, \tau)$ be $T_{2}(i)$ and $x \in X$. We denote the intersection of IC neighborhoods of $\underset{\sim}{x}$ by the IS $C=\left\langle X, C_{1}, C_{2}\right\rangle$. We assume the contrary and suppose that there exists a distinct IP $y$ in $C$, that is, $y \in C_{1}$.

CASE 1. $\{x\} \underset{\neq}{\subset} C_{1}$, then there exists $y \in C_{1}$ such that $x \neq y$. Since $(X, \tau)$ is $T_{2}(i)$, there exist IOS's $U$ and $V$ such that $\underset{\sim}{x} \in U, y \in V$, and $U \cap V=\varnothing_{\sim}$ which implies that $U \subseteq \bar{V}$. Hence we have $\underset{\sim}{x} \in U \subseteq \bar{V}$. Thus $\bar{V}$ is a closed neighborhood of $\underset{\sim}{x}$. From our assumption, we get $\underset{\sim}{y \in \bar{V}}$. But it is a contradiction, since $V_{1} \cap V_{2}=\varnothing$. Thus our assumption is false. This means that $C$ consists only of the IP $\underset{\sim}{x}$.

CASE 2. $\{x\} \underset{\neq}{\subset} C_{2}^{c}$ and $\{x\}=C_{1}$, then there exists $y \in C_{2}^{c}$ such that $y \neq x$. Since $(X, \tau)$ is $T_{2}(i)$, there exist IOS's $U, V \in \tau$ such that $\underset{\sim}{x} \in U, \underset{\sim}{y} \in V$, and $U \cap V=\varnothing_{\sim}^{\varnothing}$ and the same result as in the previous assumption holds in this case, too.

Proposition 3.25. Let $(X, \tau)$ be an ITS, $(Y, \Phi)$ a $T_{2}(i)$ ITS and $f:(X, \tau) \rightarrow(Y, \Phi)$ a continuous function. Then the graph of $f$ is an ICS in $X \times Y$.

Proof. We must show that $\overline{\operatorname{GR}(f)}$ is an IOS in $X \times Y$. Let $(x, y)_{\sim} \in \overline{\operatorname{GR}(f)}$. Then $(x, y) \in\{(x, f(x)): x \in X\}^{c}$ which implies that $y \neq f(x)$. Since $(Y, \Phi)$ is $T_{2}(i)$, there exist $U, V \in \Phi$ such that $y \in U, f \underset{\sim}{x}) \in V$, and $U \cap V=\underset{\sim}{\varnothing}$. From the assumption that $f$ is continuous, we see that $f^{-1}(V)=\left\langle X, f^{-1}\left(V_{1}\right), f^{-1}\left(V_{2}\right)\right\rangle$ is an open neighborhood of $\underset{\sim}{x}$. Also $f^{-1}(V) \times U$ is an open neighborhood of $(x, y) \sim$. It can be shown easily that $f^{-1}(V) \times U \subseteq \overline{\mathrm{GR}(f)}$. Since $\overline{\mathrm{GR}(f)}$ is not a proper IS in $X \times Y$, our assumption holds, that is, $\overline{\mathrm{GR}(f)}$ is an IOS in $X \times Y$.

Proposition 3.26. Let $(X, \tau)$ be an ITS, $(Y, \Phi)$ a $T_{2}(i)$ ITS and $f:(X, \tau) \rightarrow(Y, \Phi)$ a continuous function. Then the IS $C=\left\langle\left(x_{1}, x_{2}\right),\left\{\left(x_{1}, x_{2}\right): f\left(x_{1}\right)=f\left(x_{2}\right)\right\},\left\{\left(x_{1}, x_{2}\right)\right.\right.$ : $\left.\left.f\left(x_{1}\right) \neq f\left(x_{2}\right)\right\}\right\rangle$ in $X \times Y$ is an ICS in $X \times Y$.

Proof. A similar argument as in the proof of Proposition 3.25 can be followed.

Proposition 3.27. Let $(X, \tau)$ and $(Y, \Phi)$ be two ITS's. Then
(a) If $(X, \tau)$ and $(Y, \Phi)$ are $T_{1}(i)$, then so is $(X \times Y, \tau \times \Phi)$.
(b) If $(X, \tau)$ and $(Y, \Phi)$ are $T_{1}(i i)$, then so is $(X \times Y, \tau \times \Phi)$. 
Proof. (a) Let $(X, \tau)$ and $(Y, \Phi)$ be $T_{1}(i)$. Let $\left(x_{1}, y_{1}\right),\left(x_{2}, y_{2}\right) \in X \times Y$ and $\left(x_{1}, y_{1}\right) \neq$ $\left(x_{2}, y_{2}\right)$. Now suppose that $x_{1} \neq x_{2}$. Since $(X, \tau)$ is $T_{1}(i)$ then there exist $U, V \in \tau$ such that $x_{1} \in U, x_{2} \notin U$, and $x_{2} \in V, x_{1} \notin V$. Then we have IOS's $U \times \underset{\sim}{Y}=\left\langle(X, Y), U_{1} \times\right.$ $\left.Y,\left(U_{2}^{c} \times \varnothing^{c}\right)^{c}\right\rangle$ and $V \times \underset{\sim}{Y}=\left\langle(X, Y), V_{1} \times Y,\left(V_{2}^{c} \times \varnothing^{c}\right)^{c}\right\rangle$ in $\tau \times \Phi$ having the properties $\left(x_{1}, y_{1}\right)_{\sim} \in U \times \underset{\sim}{Y},\left(x_{2}, \tilde{y}_{2}\right)_{\sim} \notin U \times \underset{\sim}{Y}$, and $\left(x_{2}, y_{2}\right)_{\sim} \in V \times \underset{\sim}{Y},\left(x_{1}, y_{1}\right)_{\sim} \notin V \times \underset{\sim}{Y}$. We can prove the case $y_{1} \neq y_{2}$ similarly. Thus we conclude that $(X \times Y, \tau \times \Phi)$ is $T_{1}(i)$.

(b) Similar to the previous one.

Proposition 3.28. Let $(X, \tau)$ and $(y, \Phi)$ be two ITS's. Then

(a) If $(X, \tau)$ and $(Y, \Phi)$ are $T_{2}(i)$, then so is $(X \times Y, \tau \times \Phi)$.

(b) If $(X, \tau)$ and $(Y, \Phi)$ are $T_{2}(i i)$, then so is $(X \times Y, \tau \times \Phi)$.

(c) If $(X, \tau)$ and $(Y, \Phi)$ are $T_{2}(i i i)$, then so is $(X \times Y, \tau \times \Phi)$.

(d) If $(X, \tau)$ and $(Y, \Phi)$ are $T_{2}(v i i)$, then so is $(X \times Y, \tau \times \Phi)$.

Proof. (a) Let $(X, \tau),(Y, \Phi)$ be $T_{2}(i)$. Let $\left(x_{1}, y_{1}\right),\left(x_{2}, y_{2}\right) \in X \times Y$, and $\left(x_{1}, y_{1}\right) \neq$ $\left(x_{2}, y_{2}\right)$ and suppose that $x_{1} \neq x_{2}$. Since $(X, \tau)$ is $T_{2}(i)$ then there exist $U, V \in \tau$ such that $x_{1} \in U, x_{2} \in V$, and $U \cap V=\underset{\sim}{\varnothing}$. Then we can form the IOS's $U \times \underset{\sim}{Y}=\left\langle(X, Y), U_{1} \times\right.$ $\left.Y,\left(U_{2}^{c} \times \varnothing^{c}\right)^{c}\right\rangle$ and $V \times \underset{\sim}{Y}=\left\langle(X, Y), V_{1} \times Y,\left(V_{2}^{c} \times \varnothing^{c}\right)^{c}\right\rangle$ in $\tau \times \Phi$ which contains $\left(x_{1}, y_{1}\right) \sim$ and $\left(x_{2}, y_{2}\right)_{\sim}$, respectively. Now we must see that $(U \times \underset{\sim}{Y}) \cap(V \times \underset{\sim}{Y})=\underset{\sim}{\varnothing}$. Indeed,

$$
\begin{aligned}
(U \times \underset{\sim}{Y}) \cap(V \times \underset{\sim}{Y}) & =\left\langle(X, Y),\left(U_{1} \times Y\right) \cap\left(V_{1} \times Y\right),\left(U_{2}^{c} \times \varnothing^{c}\right)^{c} \cup\left(V_{2}^{c} \times \varnothing^{c}\right)^{c}\right\rangle \\
& =\left\langle(X, Y),\left(U_{1} \cap V_{1}\right) \times(Y \cap Y),\left[\left(U_{2}^{c} \times Y\right) \cap\left(V_{2}^{c} \times Y\right)\right]^{c}\right\rangle \\
& =\left\langle(X, Y), \varnothing \times Y,\left[\left(U_{2}^{c}\right) \cap\left(V_{2}^{c}\right) \times(Y \cap Y)\right]^{c}\right\rangle \\
& =\langle(X, Y), \varnothing, X \times Y\rangle={\underset{\sim}{\sim} .}^{.}
\end{aligned}
$$

Thus $(X \times Y, \tau \times \Phi)$ is $T_{2}(i)$.

(b) Similar to previous one.

(c) Assume that $(X, \tau)$ and $(Y, \Phi)$ are $T_{2}($ iii $)$. Let $\left(x_{1}, y_{1}\right),\left(x_{2}, y_{2}\right) \in X \times Y$ and $\left(x_{1}, y_{1}\right) \neq\left(x_{2}, y_{2}\right)$. Suppose that $x_{1} \neq x_{2}$. Since $(X, \tau)$ is $T_{2}(i i i)$, then there exist $U, V \in$ $\tau$ such that $x_{1} \in U, x_{2} \in V$, and $U \subseteq \bar{V}$. Then we have IOS's $U \times \underset{\sim}{Y}=\left\langle(X, Y), U_{1} \times\right.$ $\left.Y,\left(U_{2}^{c} \times \varnothing^{c}\right)^{c}\right\rangle$ and $V \underset{\sim}{Y}=\left\langle(X, Y), V_{1} \times Y,\left(V_{2}^{c} \times \varnothing^{c}\right)^{c}\right\rangle$ in $\tau \times \Phi$ containing $\left(x_{1}, y_{1}\right)_{\sim}$ and $\left(x_{2}, y_{2}\right)_{\sim}$, respectively. Now, it is easy to see that $U \times \underset{\sim}{Y} \subseteq \overline{V \times Y}$ holds, which is identical to $U_{1} \times Y \subseteq\left(V_{2}^{c} \times Y\right)^{c}$ and $V_{1} \times Y \subseteq\left(U_{2}^{c} \times Y\right)^{c}$. A similar argument holds if $y_{1} \neq y_{2}$. Thus we conclude that $(X \times Y, \tau \times \Phi)$ is $T_{2}(i i i)$.

(d) We are to show that $\Delta_{X \times Y}$ is an ICS, that is, $\bar{\Delta}_{X \times Y}$ is an IOS. Since $\bar{\Delta}_{X \times Y}$ is not a proper IS in $X \times Y$, it is sufficient to show that for every $\left(\left(p_{1}, q_{1}\right),\left(p_{2}, q_{2}\right)\right)_{\sim} \in \bar{\Delta}_{X \times Y}$, there exists an IOS $S$ in $(X \times Y) \times(X \times Y)$ such that $\left(\left(p_{1}, q_{1}\right),\left(p_{2}, q_{2}\right)\right) \sim \in S \subseteq \bar{\Delta}_{X \times Y}$. Since $\left(\left(p_{1}, q_{1}\right),\left(p_{2}, q_{2}\right)\right)_{\sim} \in \bar{\Delta}_{X \times Y}$, we get $\left(\left(p_{1}, q_{1}\right) \neq\left(p_{2}, q_{2}\right)\right)_{\sim}$, that is, $p_{1} \neq p_{2}$ or $q_{1} \neq q_{2}$. Here come three possible cases:

(1) $p_{1} \neq p_{2}, q_{1}=q_{2}$

(2) $p_{1}=p_{2}, q_{1} \neq q_{2}$

(3) $p_{1} \neq p_{2}, q_{1} \neq q_{2}$.

Here we show only case (3). Other cases can be proved similarly. Let $p_{1} \neq p_{2}$, $q_{1} \neq q_{2}$. Since $\left(p_{1}, p_{2}\right)_{\sim} \in \bar{\Delta}_{X},\left(q_{1}, q_{2}\right)_{\sim} \in \bar{\Delta}_{Y}$ and $\bar{\Delta}_{X}, \bar{\Delta}_{Y}$ are IOS's, $\exists U_{1}, U_{2} \in \tau$ and $V_{1}$, 
$V_{2} \in \Phi$ such that $\left(p_{1}, p_{2}\right)_{\sim} \in U_{1} \times U_{2} \subseteq \bar{\Delta}_{X}$ and $\left(q_{1}, q_{2}\right)_{\sim} \in V_{1} \times V_{2} \subseteq \bar{\Delta}_{Y}$. We prove that $\left(\left(p_{1}, q_{1}\right),\left(p_{2}, q_{2}\right)\right)_{\sim} \in\left(U_{1} \times V_{1}\right) \times\left(U_{2} \times V_{2}\right) \subseteq \bar{\Delta}_{X \times Y}$. This can be shown in two steps.

STEP 1. The expression $\left(\left(p_{1}, q_{1}\right),\left(p_{2}, q_{2}\right)\right)_{\sim} \in\left(U_{1} \times V_{1}\right) \times\left(U_{2} \times V_{2}\right)$ is equivalent to $\left(\left(p_{1}, q_{1}\right),\left(p_{2}, q_{2}\right)\right) \in\left(U_{1} \times V_{1}\right)^{(1)} \times\left(U_{2} \times V_{2}\right)^{(1)} \Leftrightarrow\left(\left(p_{1}, q_{1}\right),\left(p_{2}, q_{2}\right)\right) \in\left(U_{1}^{(1)} \times V_{1}^{(1)}\right) \times$ $\left(U_{2}^{(1)} \times V_{2}^{(1)}\right)$. This means that $\left(p_{1}, q_{1}\right) \in U_{1}^{(1)} \times V_{1}^{(1)}$ and $\left(p_{2}, q_{2}\right) \in U_{2}^{(1)} \times V_{2}^{(1)}$ which are true, since $p_{1} \in U_{1}^{(1)}, p_{2} \in U_{2}^{(1)}, q_{1} \in V_{1}^{(1)}, q_{2} \in V_{2}^{(1)}$.

STEP 2. We show the inclusion $\left(U_{1} \times V_{1}\right) \times\left(U_{2} \times V_{2}\right) \subseteq \bar{\Delta}_{X \times Y}$. For this purpose we must first show that $\left(U_{1} \times V_{1}\right)^{(1)} \times\left(U_{2} \times V_{2}\right)^{(1)} \subseteq\left\{\left(\left(u_{1}, v_{1}\right),\left(u_{2}, v_{2}\right)\right):\left(u_{1}, v_{1}\right) \neq\right.$ $\left.\left(u_{2}, v_{2}\right)\right\}$ or equivalently, $\left(U_{1}^{(1)} \times V_{1}^{(1)}\right) \times\left(U_{2}^{(1)} \times V_{2}^{(1)}\right) \subseteq\left\{\left(\left(u_{1}, v_{1}\right),\left(u_{2}, v_{2}\right)\right):\left(u_{1}, v_{1}\right) \neq\right.$ $\left.\left(u_{2}, v_{2}\right)\right\}$. This is true since $U_{1} \times U_{2} \subseteq \bar{\Delta}_{X}$ and $V_{1} \times V_{2} \subseteq \bar{\Delta}_{Y}$, we have $U_{1}^{(1)} \times U_{2}^{(1)} \subseteq$ $\left\{\left(u_{1}, u_{2}\right): u_{1} \neq u_{2}\right\}$ and $V_{1}^{(1)} \times V_{2}^{(1)} \subseteq\left\{\left(v_{1}, v_{2}\right): v_{1} \neq v_{2}\right\}$, respectively. Thus the first inclusion is true. The second inclusion can be proved similarly. Hence $\bar{\Delta}_{X \times Y}$ is an IOS, that is, $\bar{\Delta}_{X \times Y}$ is an ICS, which means that $(X, Y, \tau \times \Phi)$ is $T_{2}(v i i)$.

REMARK 3.29. Let $(X, \tau)$ and $(Y, \Phi)$ be $T_{2}(i v)$. Then $(X \times Y, \tau \times \Phi)$ may not be $T_{2}(i v)$.

Here come the reverse implications.

Proposition 3.30. Let $(X, \tau)$ and $(Y, \Phi)$ be two ITS's. Then

(a) If $(X \times Y, \tau \times \Phi)$ is $T_{2}(i)$, then so are $(X, \tau)$ and $(Y, \Phi)$.

(b) If $(X \times Y, \tau \times \Phi)$ is $T_{2}(i i)$, then so are $(X, \tau)$ and $(Y, \Phi)$.

(c) If $(X \times Y, \tau \times \Phi)$ is $T_{2}(i i i)$, then so are $(X, \tau)$ and $(Y, \Phi)$.

Proof. The proofs of (a) and (b) are easy. (c) Let $(X \times Y, \tau \times \Phi)$ be $T_{2}$ (iii), and $x_{1} \neq x_{2}\left(x_{1}, x_{2} \in X\right)$. We take a fixed $y \in Y$. Then, since $\left(x_{1}, y\right) \neq\left(x_{2}, y\right)$ and $X \times Y$ is $T_{2}$ (iii), there exist $U \times Z$ and $V \times T$ where $U, V \in \tau$ and $Z, T \in \Phi$ such that $\left(x_{1}, y\right)_{\sim} \in$ $U \times Z,\left(x_{2}, y\right)_{\sim} \in V \times T$, and $U \times Z \subseteq \overline{V \times T}$. Thus we get $\left(x_{1}, y\right) \in U_{1} \times Z_{1},\left(x_{2}, y\right) \in$ $V_{1} \times T_{1}$, and $U_{1} \times Z_{1} \subseteq\left(V_{2}^{c} \times T_{2}^{c}\right)^{c}, V_{1} \times T_{1} \subseteq\left(U_{2}^{c} \times Z_{2}^{c}\right)^{c}$; in other words $x_{1} \in U_{1}, y \in Z_{1}$, $x_{2} \in V_{1}, y \in T_{1}$, and $\left(U_{1} \times Z_{1}\right) \cap\left(V_{2}^{c} \times T_{2}^{c}\right)=\varnothing,\left(V_{1} \times T_{1}\right) \cap\left(U_{2}^{c} \times Z_{2}^{c}\right)=\varnothing$. From the last intersection we get $\left(U_{1}^{c} \times V_{2}^{c}\right) \times\left(Z_{1} \cap T_{2}^{c}\right)=\varnothing$ and $\left(V_{1} \cap U_{2}^{c}\right) \times\left(T_{1} \cap Z_{2}^{c}\right)=\varnothing$, respectively. $y \in Z_{1}$ and $y \in T_{1}$ implies that $Z_{1} \cap T_{2}^{c} \neq \varnothing$ and $U_{1} \cap V_{2}^{c}=\varnothing$ from which $U_{1} \subseteq V_{2}$ follows. Similarly $y \in T_{1} \cap Z_{2}^{c}$ and $V_{1} \cap U_{2}^{c}=\varnothing$ meaning that $V_{1} \subseteq U_{2}$. Thus $x_{\sim} \in U, \underset{\sim}{x_{2}} \in V$, and $U \subseteq \bar{V}$, that is, $(X, \tau)$ is $T_{2}$ (iii). Similarly $(Y, \Phi)$ is $T_{2}(i i i)$, too.

\section{REFERENCES}

[1] K. Atanassov, Intuitionistic fuzzy sets, VII ITKR's Session (Sofia, June 1983 Central Sci. and Tech. Library) (V. Sgurev, ed.), Blug. Academy of Sciences, Sofia, 1984.

[2] _ Intuitionistic fuzzy sets, Fuzzy Sets and Systems 20 (1986), no. 1, 87-96. MR 87f:03151. Zbl 631.03040.

[3] S. Bayhan and D. Çoker, On fuzzy separation axioms in intuitionistic fuzzy topological spaces, BUSEFAL 67 (1996), 77-87.

[4] D. Çoker, A note on intuitionistic sets and intuitionistic points, Turkish J. Math. 20 (1996), no. 3, 343-351. MR 99c:03100. Zbl 862.04007.

[5] _ An introduction to intuitionistic fuzzy topological spaces, Fuzzy Sets and Systems 88 (1997), no. 1, 81-89. MR 97m:54009. Zbl 923.54004.

[6] _ An introduction to intuitionistic topological spaces, BUSEFAL 81 (2000), 51-56.

[7] D. Çoker and M. Demirci, On intuitionistic fuzzy points, Notes IFS 1 (1995), no. 2, 79-84. CMP 1417 217. Zbl 850.04011. 
[8] D. Çoker and A. H. Eş, On fuzzy compactness in intuitionistic fuzzy topological spaces, J. Fuzzy Math. 3 (1995), no. 4, 899-909. MR 96j:54010. Zbl 846.54003.

[9] E. Coşkun and D. Çoker, On neighborhood structures in intuitionistic topological spaces, Math. Balkanica (N.S.) 12 (1998), no. 3-4, 283-293. MR 1688 660. Zbl 01505530.

[10] A. A. Fora, Separation axioms for fuzzy spaces, Fuzzy Sets and Systems 33 (1989), no. 1, 59-75. MR 90k:54011. Zbl 702.54007.

[11] M. H. Ghanim, E. E. Kerre, and A. S. Mashhour, Separation axioms, subspaces and sums in fuzzy topology, J. Math. Anal. Appl. 102 (1984), no. 1, 189-202. MR 86i:54005. Zbl 543.54006.

[12] S. Özçağ and D. Çoker, On connectedness in intuitionistic fuzzy special topological spaces, Int. J. Math. Math. Sci. 21 (1998), no. 1, 33-40. CMP 1486 955. Zbl 892.54005.

[13] R. Srivastava, S. N. Lal, and A. K. Srivastava, Fuzzy Hausdorff topological spaces, J. Math. Anal. Appl. 81 (1981), no. 2, 497-506. MR 83j:54005. Zbl 491.54004.

[14] _ Fuzzy T $T_{1}$-topological spaces, J. Math. Anal. Appl. 102 (1984), no. 2, 442-448. MR 85m:54008. Zbl 557.54003.

[15] L. A. Zadeh, Fuzzy sets, Information and Control 8 (1965), 338-353. MR 36\#2509. Zbl 0139.24606.

SADik Bayhan: Department of Mathematics, HacetTePe University, Beytepe, 06532 ANKARA, TURKEY

DoĞAN ÇOKER: Department of MATHEMATICs, AKDENiz University, 07058 ANTALya, TURKEY 


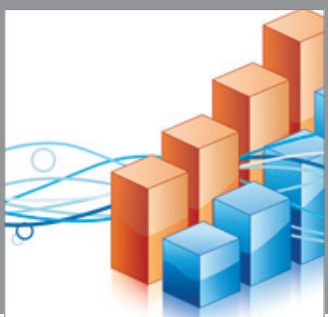

Advances in

Operations Research

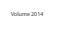

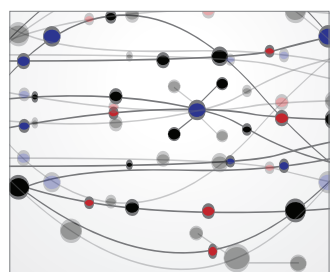

\section{The Scientific} World Journal
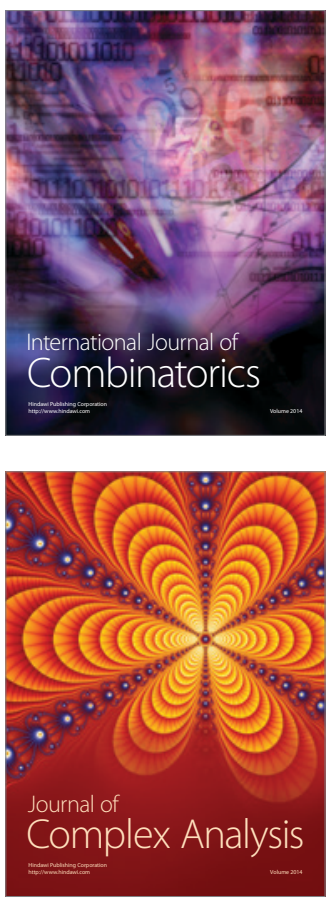

International Journal of

Mathematics and

Mathematical

Sciences
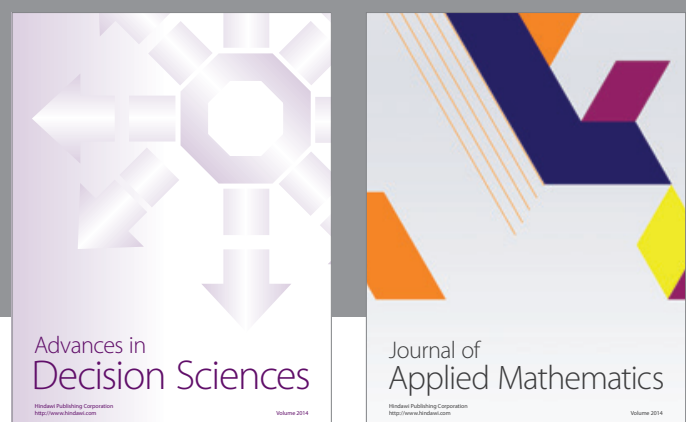

Journal of

Applied Mathematics
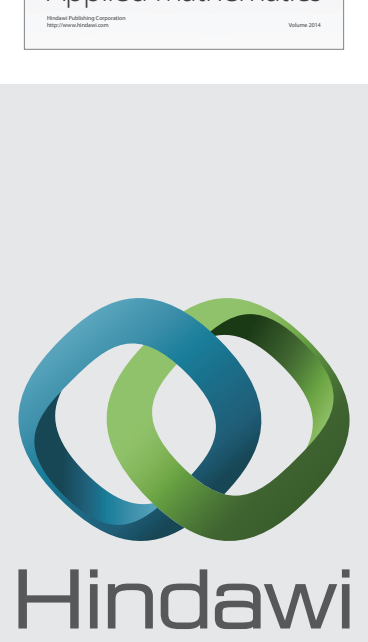

Submit your manuscripts at http://www.hindawi.com
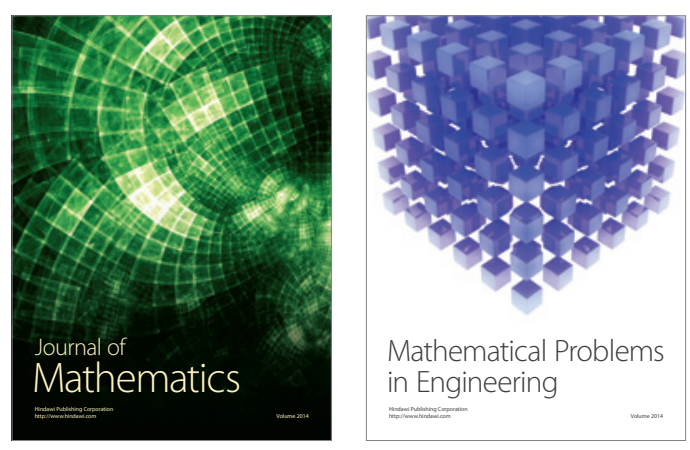

Mathematical Problems in Engineering
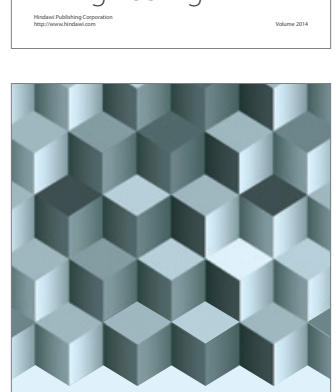

Journal of

Function Spaces
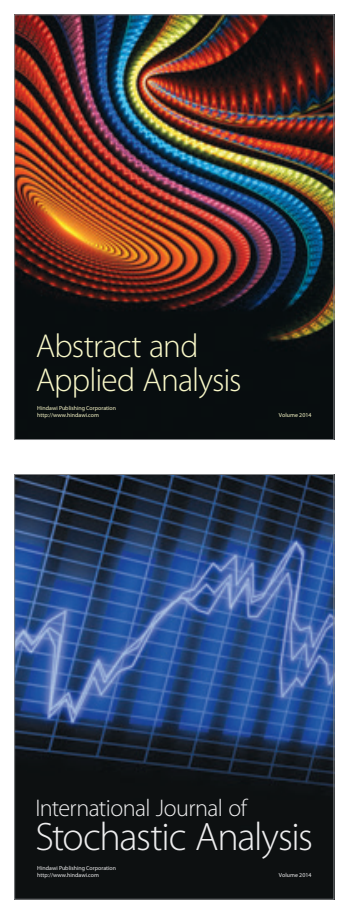

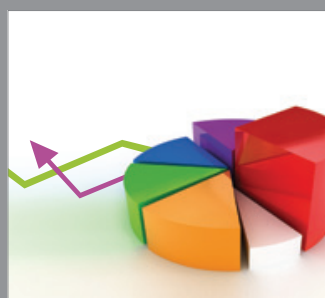

ournal of

Probability and Statistics

Promensencen
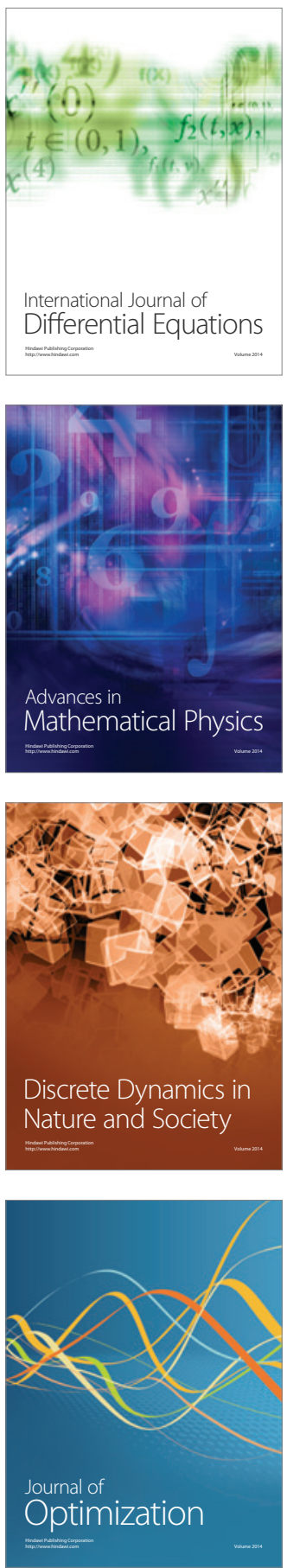